\title{
Combined duodenal stenting and endoscopic ultrasound-guided hepatico- gastrostomy using forward-viewing echoendoscope: a one-scope technique
}
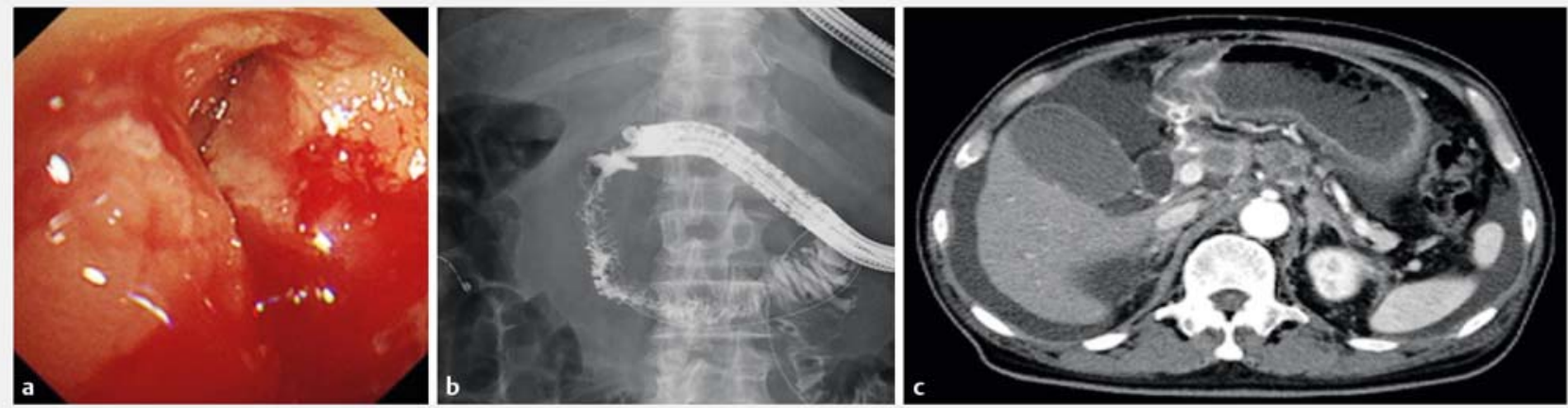

- Fig. 1 Views of the cancer and obstruction. a Endoscopic image of distal gastric cancer. b Fluoroscopic image of duodenal stricture. c Computed tomography of abdomen showing hilar lymph node metastasis.

Gastric cancer is the second leading cause of cancer mortality all over the world. Although early detection has increased the curative resection, many cases still present in an advanced stage with gastric outlet obstruction and lymph node metastasis $[1,2]$. Here, we report a novel technique for palliative management of pyloric obstruction and obstructive jaundice in the same session using a forwardviewing echoendoscope.

A 71-year-old patient with advanced gastric cancer presented with pyloric obstruction and hilar obstructive jaundice ( Fig.1). We decided on palliative management including duodenal stenting and an endoscopic ultrasound (EUS)guided hepaticogastrostomy using a forward-viewing echoendoscope.

First, we marked the esophagogastric junction with an endoclip to avoid transesophageal puncture. We advanced the forward-viewing echoendoscope down to the gastric antrum. Under fluoroscopic guidance, the stricture site was determined and then cannulated with a 0.25 -inch guidewire within the catheter ( Fig. 1). A $2.2-\mathrm{cm} \times 12-\mathrm{cm}$ uncovered duodenal stent (HANAROSTENT; Olympus, Tokyo, Japan) was advanced over the guidewire traversing the stricture site, both ends of the stent were care-
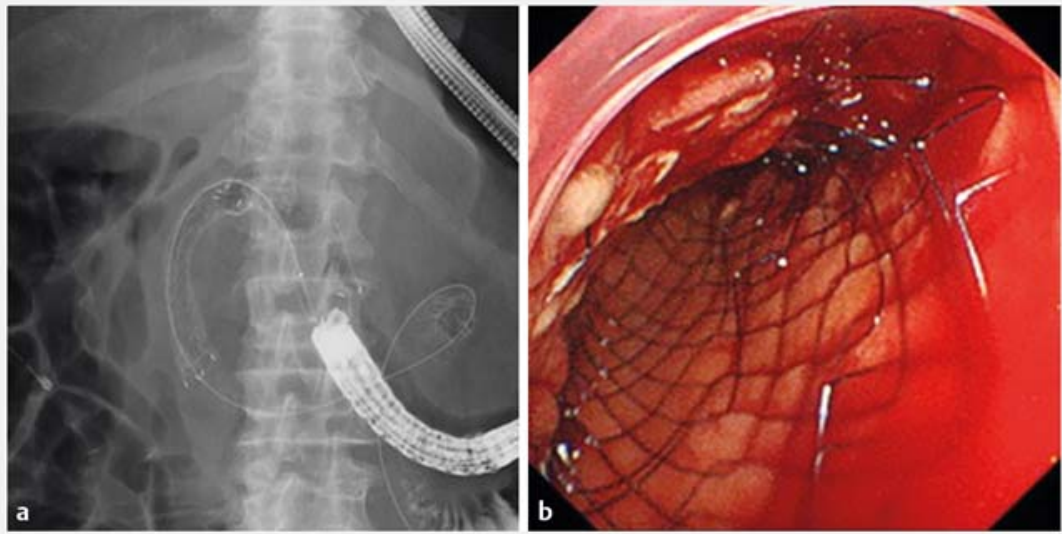

- Fig.2 Fluoroscopic and endoscopic images showing deployed duodenal stent.

fully monitored, and then the stent was successfully deployed ( $\bullet$ Fig. 2 ).

Second, we visualized the liver and determined the bile duct of segment 2 (B2) with the same endoscope. We punctured B2 using a 22-gauge needle (Expect Slimline (SL); Boston Scientific, Marlborough, Massachusetts) with a preloaded 0.018-inch guidewire ( $>$ Fig.3). After bile duct confirmation by contrast medium, we dilated the track by using a 7- $\mathrm{Fr}$ mechanical dilator (ES dilator; ZEON Medical, Tokyo, Japan). We successfully deployed the hepaticogastrostomy stent
(5.9-Fr delivery system, HANAROSTENT Benefit, $6 \mathrm{~mm} \times 10 \mathrm{~cm}$, fully covered; Boston Scientific) (> Fig.4).

Here, we recommend a forward-viewing echoendoscope for both duodenal stenting and EUS-guided hepaticogastrostomy. With a wide working channel and forward-view orientation, this endoscope allows the output of the duodenal stent easily over the guidewire. Moreover, it enabled us to perform the hepaticogastrostomy at the same time. In comparison to the curved echoendoscope, the forward-viewing echoendoscope 

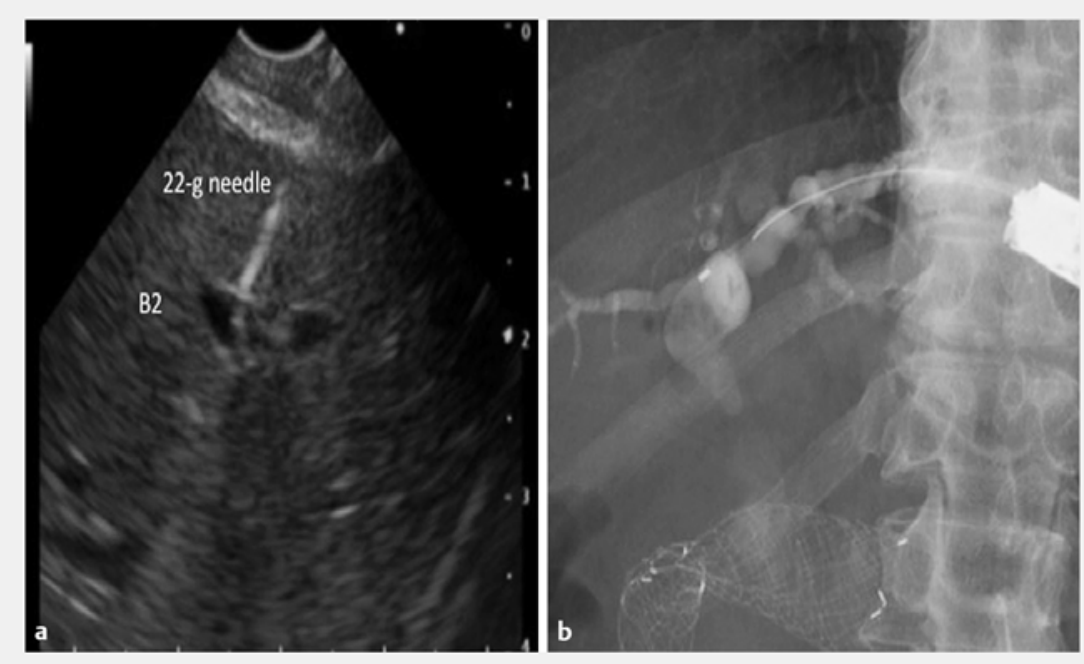

Fig. 3 Views of the procedure. a Endoscopic-ultrasound image of the left lobe of the liver showing B2. b Fluoroscopic image of B2 puncture and cannulation with forward-viewing echoendoscope.

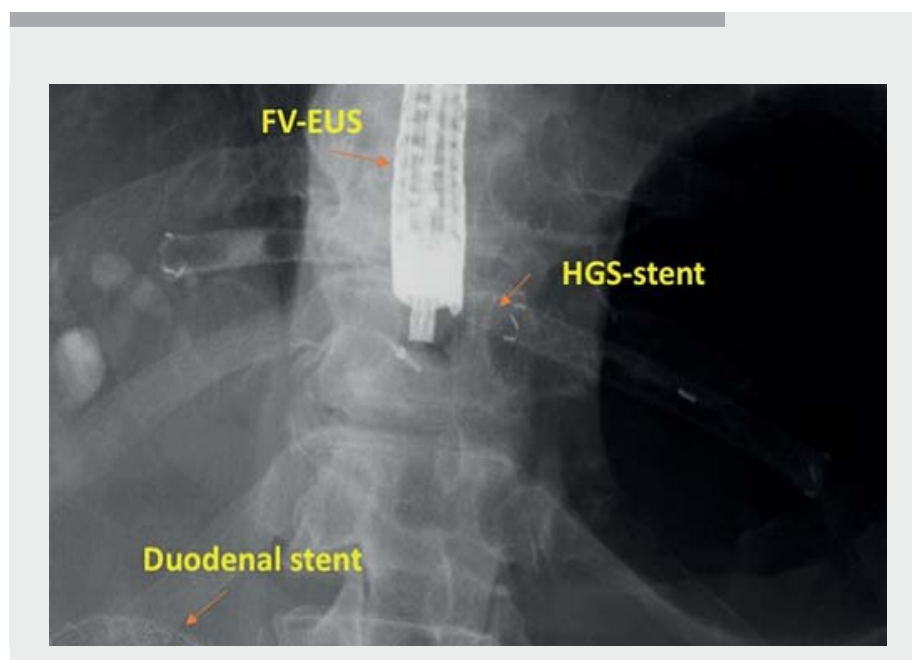

Video 1 How to perform duodenal stenting and endoscopic ultrasound-guided hepaticogastrostomy using only a forward-viewing echoendoscope.

makes transgastric B2-puncture easier because of the forward-view orientation [3]. We successfully deployed the duodenal stent and performed the hepaticogastrostomy easily using only a forwardviewing echoendoscope ( $\vee$ Video 1$)$.

\section{Endoscopy_UCTN_Code_CPL_1AH_2AD}

\section{Competing interests}

The authors declare that they have no conflict of interest.

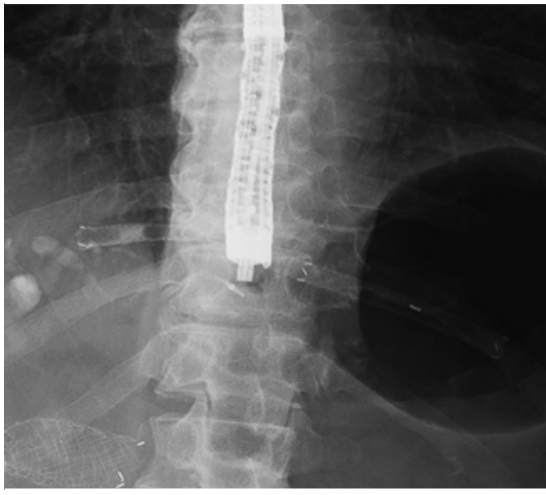

-Fig. 4 Fluoroscopic image of both duodenal and hepaticogastrostomy stents.

\section{Corresponding author}

\section{Kazuo Hara, MD}

Department of Gastroenterology, Aichi Cancer Center Hospital, 1-1 Kanokoden, Chikusa-ku, Nagoya, Aichi 464-8681, Japan Fax: +81-52-7635233

khara@aichi-cc.jp

\section{References}

[1] Endo S, Takiguchi S, Miyazaki Y et al. Efficacy of endoscopic gastroduodenal stenting for gastric outlet obstruction due to unresectable advanced gastric cancer: a prospective multicenter study. J Surg Oncol 2014; 109 : 208-212

[2] Maruyama K, Gunvén P, Okabayashi K et al. Lymph node metastases of gastric cancer. General pattern in 1931 patients. Ann Surg 1989; 210: 596-602

[3] Hara K, Okuno N, Haba S et al. How to perform EUS-guided hepaticogastrostomy easier and safer. J Hepatobiliary Pancreat Sci 2020; 27: 563-564

\section{Bibliography}

Endoscopy 2022; 54: E516-E517

DOI $10.1055 / \mathrm{a}-1640-8177$

ISSN 0013-726X

published online 25.10 .2021

(c) 2021. Thieme. All rights reserved.

Georg Thieme Verlag KG, Rüdigerstraße 14, 70469 Stuttgart, Germany 